2 Ammann AJ, Wara DW, Dritz S, et al. Acquired immuno deficiency syndrome in an infant: possible transmission by means of blood products. Lancet $1983 ;$ i :956-8.

${ }^{3}$ Lederman MM, Ratnoff OD, Scillian JJ, Jones PK, Schacter B. Impaired cell mediated immunity in patients with classical haemophilia. $N$ Englf Med 1983;308:79-83.

4 Menitove JE, Asher RH, Casper JT, et al. T-lymphocyte subpopulations in patients with classic haemophilia treated with cryoprecipitate and lyophilised concentrates. N Engl f Med 1983;308:83-6.

${ }^{5}$ Reinherz EI, Schlossman SF. The differentiation and function of human T lymphocytes. Cell 1980;19:821-7.

${ }^{6}$ Kay HD, Fagnani R, Bonnard GD. Cytotoxicity against the K562 erythroleukaemia cell line by human natural killer (NK) cells which do not bear free FC receptors for IgG. Int $\mathcal{F}$ Cancer 1979;24:141-50.

${ }^{7}$ Froebel K, Sturrock RD, Reynolds P, Grennan A, Roxburgh A, MacSween RNM. In-vitro reactions of lymphocytes in rheumatoid arthritis and other rheumatic diseases. Ann Rheum Dis 1979;38:535-43.

" Ryan TA Jr, Joiner BL, Ryan BF. Minitab reference manual. Boston: Duxbury Press, 1981.
${ }^{9}$ Gordon RS. Factor VIII products and disordered immune regulation. Lancet 1983;i:991.

10 Ludlam CA, Carr R, Veitch SE, Steel CM. Disordered immune regulation in haemophiliacs not exposed to commercial factor VIII. Lancet 1983; i : 1226 .

${ }^{11}$ Ragni MV, Spero JA, Lewis JH, Bontempo FA. Acquired immune deficiency-like syndrome in two haemophiliacs. Lancet 1983;i:213-4.

12 Goldsmith JC, Moseley PL, Monick M, Brady M, Hunninghake GW T-lymphocyte subpopulation abnormalities in apparently healthy patients with haemophilia. Ann Intern Med 1983;98:294-6.

13 Friedman-Kien AE, Laubenstein LJ, Rubenstein P, et al. Disseminated Kaposi's sarcoma in homosexual man. Ann Intern Med 1982;96:693-700.

14 Vieira J, Frank E, Spira TJ, Landesman SH. Acquired immune deficiency in Haitians. $N$ Engl f Med 1983;398:125-9.

${ }^{15}$ Carney WP, Rubin RH, Hoffman RA, Hausen WP, Healey K, Hirsch MS. Analysis of $\mathrm{T}$ lymphocyte subsets in cytomegalovirus mononucleosis. $\mathcal{F}$ Immunol $1981 ; 126: 2114-6$.

(Accepted 25 August 1983)

\title{
Gonadal function in men treated for acute leukaemia
}

\author{
J WAXMAN, Y TERRY, L H REES, T A LISTER
}

\begin{abstract}
Gonadal function was assessed in eight men in remission of leukaemia who had completed treatment eight months to eight years previously. All four men treated for acute myeloid leukaemia had normal sperm counts and motility, compared with only one of the four with acute lymphatic or undifferentiated leukaemia. Hormonal studies indicated that the sterility resulted from gonadal failure rather than pituitary dysfunction after cranial irradiation.
\end{abstract}

These findings are important in the counselling of patients with leukaemia.

\section{Introduction}

Complete remission may now be induced in most young adults with acute leukaemia, and the proportion continuing without recurrence at five years is roughly one third. Thereafter relapse is rare and cure must be considered to be probable. As with other malignancies for which the rate of cure is appreciable, attention must be given to long term complications. We performed this study to assess gonadal function after the treatment of acute leukaemia in adults.

\section{Patients and methods}

We investigated eight of 21 men aged under 50 in remission of leukaemia who had completed treatment. Their mean age at presentation was $23 \cdot 6$ years (range 15 to 33 years) and at follow up $29 \cdot 2$ years

\footnotetext{
Imperial Cancer Research Fund Department of Medical Oncology, St Bartholomew's Hospital, London EC1A 7BE

I WAXMAN, BSC, MRCP, Imperial Cancer Research Fund research fellow and honorary senior registrar

Y TERRY, SRN, BSC, sociologist

L H REES, FRCP, $M D$, PHD, professor of chemical endocrinology

T A LISTER, MD, FRCP, reader in medical oncology

Correspondence to: Dr J Waxman.
}

(range 20 to 38 years). The treatment free interval ranged from eight months to eight years. Table I gives details of treatment.

Seminal analysis was performed after a minimum of three days'

TABLE I-Details of treatment

\begin{tabular}{|c|c|c|c|}
\hline $\begin{array}{l}\text { Case } \\
\text { No }\end{array}$ & Leukaemia & $\begin{array}{l}\text { Induction agents and } \\
\text { total dosage }\end{array}$ & $\begin{array}{l}\text { Maintenance agents } \\
\text { and dosage }\end{array}$ \\
\hline 1 & $\begin{array}{l}\text { Acute } \\
\text { myeloid }\end{array}$ & $\begin{array}{l}\text { Adriamycin } 620 \mathrm{mg} \\
\text { Cytarabine } 5.6 \mathrm{~g} \\
\text { Thioguanine } 16.8 \mathrm{~g}\end{array}$ & \\
\hline 2 & $\begin{array}{l}\text { Acute } \\
\text { myeloid }\end{array}$ & $\begin{array}{l}\text { Daunorubicin } 1.68 \mathrm{~g} \\
\text { Cytarabine } 27.6 \mathrm{~g} \\
\text { Thioguanine } 9 \cdot 2 \mathrm{~g}\end{array}$ & \\
\hline 3 & $\begin{array}{l}\text { Acute } \\
\text { myeloid }\end{array}$ & $\begin{array}{l}\text { Adriamycin } 900 \mathrm{mg} \\
\text { Cytarabine } 15 \cdot 18 \mathrm{~g} \\
\text { Thioguanine } 15 \cdot 18 \mathrm{~g}\end{array}$ & \\
\hline 4 & $\begin{array}{l}\text { Acute } \\
\text { myeloid }\end{array}$ & $\begin{array}{l}\text { Adriamycin } 900 \mathrm{mg} \\
\text { Cytarabine } 8.4 \mathrm{~g} \\
\text { Thioguanine } 13.4 \mathrm{~g}\end{array}$ & \\
\hline $5^{*}$ & $\begin{array}{l}\text { Acute } \\
\text { lymphatic }\end{array}$ & $\begin{array}{l}\text { Vincristine } 12 \mathrm{mg} \\
\text { Adriamycin } 270 \mathrm{mg} \\
\text { Colaspase } 210 \times 10^{3} \mathrm{IU}\end{array}$ & $\begin{array}{l}\text { Cyclophosphamide } 18.75 \mathrm{~g} \\
\text { Methotrexate } 1.875 \mathrm{~g} \\
\text { Mercaptopurine } 43.75 \mathrm{~g}\end{array}$ \\
\hline 6* & $\begin{array}{l}\text { Acute } \\
\text { lymphatic }\end{array}$ & $\begin{array}{l}\text { Vincristine } 8 \mathrm{mg} \\
\text { Adriamycin } 200 \mathrm{mg} \\
\text { Colaspase } 16.5 \times 10^{3} \mathrm{IU}\end{array}$ & $\begin{array}{l}\text { Cyclophosphamide } 22.5 \mathrm{~g} \\
\text { Methotrexate } 2.34 \mathrm{~g} \\
\text { Mercaptopurine } 52.5 \mathrm{~g}\end{array}$ \\
\hline $7^{*}$ & $\begin{array}{l}\text { Acute } \\
\text { lymphatic }\end{array}$ & $\begin{array}{l}\text { Vincristine } 12 \mathrm{mg} \\
\text { Adriamycin } 640 \mathrm{mg} \\
\text { Colaspase } 252 \times 10^{3} \mathrm{IU} \\
\text { Cyclophosphamide } 3.6 \mathrm{~g}\end{array}$ & $\begin{array}{l}\text { Cyclophosphamide } 4.5 \mathrm{~g} \\
\text { Methotrexate } 4.5 \mathrm{~g} .5 .75 \mathrm{~g} \\
\text { Mercaptopurine } 78.75\end{array}$ \\
\hline $8^{*}$ & $\begin{array}{l}\text { Acute } \\
\text { undifferentiated }\end{array}$ & $\begin{array}{l}\text { Vincristine } 8 \mathrm{mg} \\
\text { Adriamycin } 200 \mathrm{mg} \\
\text { Colaspase } 102 \times 10^{3} \mathrm{IU}\end{array}$ & $\begin{array}{l}\text { Cyclophosphamide } 18.75 \mathrm{~g} \\
\text { Methotrexate } 2.5 \mathrm{~g} . \\
\text { Mercaptopurine } 43.5 \mathrm{~g}\end{array}$ \\
\hline
\end{tabular}

*Cranial irradiation of 2400 rads given.

abstinence. At 9 am, after basal blood specimens had been obtained for estimation of concentrations of prolactin (mean of three measurements), sex hormone binding globulin, testosterone, and $17 \beta-$ oestradiol, a standard test was performed in which luteinising hormone releasing hormone $(100 \mu \mathrm{g})$ was given. Follicle stimulating hormone, luteinising hormone, and prolactin concentrations were measured by specific double antibody radioimmunoassay using Medical Research Council standards $69 / 104,68 / 40$, and $71 / 222$ respectively. After ether extraction testosterone and oestradiol concentrations were measured by tritiated radioimmunoassay. Progesterone concentration was measured by tritiated radioimmunoassay after hexane extraction. Concentrations of sex hormone binding globulin were measured by saturation radioimmunoassay. ${ }^{1}$ 


\section{Results}

Seminal analysis (table II)-All patients treated for acute myeloid leukaemia had normal sperm counts (mean count $180 \times 10^{6} \mathrm{sperm} /$ ejaculate, range $57-253 \times 10^{6} \mathrm{sperm} /$ ejaculate) and motility. Only one of four patients treated for acute lymphatic or undifferentiated leukaemia had a normal sperm count $\left(50 \times 10^{6} \mathrm{sperm} / \mathrm{ejaculate}\right)$, the rest being azoospermic. Active spermatogenesis was unrelated to the treatment free interval. Six conceptions occurred after chemotherapy: four were congenitally normal (although one child had severe epilepsy), one resulted in a spontaneous abortion, and one resulted in a child with anencephaly. No conceptions were achieved by patients treated for acute lymphatic or undifferentiated leukaemia.

TABLE II-Results of seminal analysis

\begin{tabular}{|c|c|c|c|c|c|}
\hline \multirow{2}{*}{$\begin{array}{l}\text { Case } \\
\text { No }\end{array}$} & \multirow{2}{*}{ Leukaemia } & \multicolumn{2}{|c|}{$\begin{array}{l}\text { No of children } \\
\text { (and any abnormality) }\end{array}$} & \multirow{2}{*}{$\begin{array}{c}\text { No of } \\
\text { spontaneous } \\
\text { abortions } \\
\text { after } \\
\text { treatment }\end{array}$} & \multirow{2}{*}{$\begin{array}{l}\text { Sperm } \\
\text { count } \\
\left(\times 10^{6}\right)\end{array}$} \\
\hline & & $\begin{array}{c}\text { Before } \\
\text { treatment }\end{array}$ & $\begin{array}{l}\text { After } \\
\text { treatment }\end{array}$ & & \\
\hline 1 & Acute myeloid & 0 & 1 & 0 & 187 \\
\hline 2 & Acute myeloid & 0 & $\begin{array}{c}2 \\
\text { (1 anencephaly, }\end{array}$ & 0 & $57 \cdot 6$ \\
\hline 3 & Acute myeloid & 1 & 0 & 1 & 225 \\
\hline 4 & Acute myeloid & 1 & 2 & 0 & 253 \\
\hline 5 & Acute lymphatic & 0 & 0 & 0 & 0 \\
\hline 6 & Acute lymphatic & 0 & 0 & 0 & 50 \\
\hline 7 & Acute lymphatic & 1 & 0 & 0 & 0 \\
\hline 8 & Acute undifferentiated & 0 & 0 & 0 & 0 \\
\hline
\end{tabular}

Hormonal changes (table III)-All patients with normal sperm counts had normal or marginally raised basal gonadotrophin concentrations. In each of them a subnormal response of luteinising hormone and an exaggerated response of follicle stimulating hormone followed

TABLE III-Hormonal concentration

\begin{tabular}{|c|c|c|c|c|c|}
\hline & \multicolumn{2}{|c|}{$\begin{array}{l}\text { Acute myeloid } \\
\text { leukaemia }\end{array}$} & \multicolumn{2}{|c|}{$\begin{array}{c}\text { Acute lymphatic and } \\
\text { acute undifferentiated } \\
\text { leukaemia }\end{array}$} & \multirow[t]{2}{*}{$\begin{array}{l}\text { Normal } \\
\text { range }\end{array}$} \\
\hline & Range & Mean & Range & Mean & \\
\hline \multicolumn{6}{|l|}{$\begin{array}{l}\text { Luteinising } \\
\text { hormone }(\mathrm{U} / \mathrm{L}) \text { : }\end{array}$} \\
\hline $\begin{array}{l}\text { Baseline } \\
\text { Time after } \\
100 \mu \mathrm{g} \mathrm{LHRH:}\end{array}$ & $2 \cdot 5-5 \cdot 3$ & 3.9 & $7 \cdot 6-30$ & $18 \cdot 0$ & $1 \cdot 4-9 \cdot 7$ \\
\hline $\begin{array}{l}20 \mathrm{mins} \\
60 \mathrm{mins}\end{array}$ & $\begin{array}{l}8 \cdot 4-10 \cdot 7 \\
9 \cdot 4-10 \cdot 5\end{array}$ & $9 \cdot 4$ & $\begin{array}{l}15 \cdot 6-45 \\
14 \cdot 2-52\end{array}$ & $29 \cdot 4$ & $13 \cdot 1-57 \cdot 6$ \\
\hline \multicolumn{6}{|l|}{$\begin{array}{l}\text { Follicle stimulating } \\
\text { hormone }(\mathrm{C} L) \text { : }\end{array}$} \\
\hline $\begin{array}{l}\text { hormone }(\mathrm{C} L): \\
\text { Baseline }\end{array}$ & $4 \cdot 2-10 \cdot 8$ & 68 & $5 \cdot 2-15$ & $8 \cdot 3$ & $1-7$ \\
\hline \multicolumn{6}{|l|}{ Time after } \\
\hline $\begin{array}{l}100 \mu \mathrm{g} \text { LHRH: } \\
20 \mathrm{mins}\end{array}$ & $47-53$ & 51 & $27 \cdot 6-62$ & $41 \cdot 3$ & $1-7$ \\
\hline 60 mins & $37 \cdot 5-50$ & 44 & $19-77$ & $40 \cdot 4$ & $0 \cdot 8-5 \cdot 2$ \\
\hline Progesterone (nmol/1) & $3 \cdot 7-8 \cdot 7$ & $6 \cdot 2$ & $3 \cdot 2-7 \cdot 7$ & $5 \cdot 2$ & $<12$ \\
\hline $\begin{array}{l}\text { 17ß-Oestradiol } \\
(\mathrm{pmol} 1)\end{array}$ & $130-200$ & 175 & $130-200$ & 155 & $<30-128$ \\
\hline $\begin{array}{l}\text { Sex hormone binding } \\
\text { globulin }(\mathrm{nmol} / 1)\end{array}$ & $24-41$ & 32 & $21-51$ & $30 \cdot 9$ & $17-50$ \\
\hline Testosterone $(\mathrm{nmol} / \mathrm{l})$ & $8-34$ & $19 \cdot 3$ & $13 \cdot 3-19$ & $16 \cdot 3$ & $10-38$ \\
\hline $\begin{array}{l}\text { Mean prolactin } \\
(\mathrm{mU} / 1)\end{array}$ & $115-246$ & 170 & $263-391$ & 336 & $<360$ \\
\hline
\end{tabular}

LHRH $=$ Luteinising hormone releasing hormone.

Conversion: SI to traditional units-Progesterone: $1 \mathrm{nmol} / 1 \approx 0.31 \mathrm{ng} / \mathrm{ml} .17 \beta-$ Oestradiol: $1 \mathrm{pmol} / 1 \approx 0.27 \mathrm{pg} / \mathrm{ml}$. Sex hormone binding globulin: $1 \mathrm{nmol} / 1 \approx 0.05$ $\mu \mathrm{g} / \mathrm{ml}$. Testosterone: $1 \mathrm{nmol} / 1 \approx 0 \cdot 29 \mathrm{ng} / \mathrm{ml}$.

injection of $100 \mu \mathrm{g}$ luteinising hormone releasing hormone. Two of the three patients with azoospermia had raised basal serum gonadotrophin concentrations; all three had normal dynamic responses of luteinising hormone but exaggerated responses of follicle stimulating hormone to the injection of luteinising hormone releasing hormone. All of the patients had normal or marginally raised serum concentrations of progesterone, sex hormone binding globulin, and 17\%-oestradiol. Two patients, who had been treated for acute lymphatic and acute undifferentiated leukaemia, had marginally raised serum prolactin concentrations. All but one patient had normal serum testosterone concentrations.

\section{Discussion}

These results show that relatively short term cyclical combination chemotherapy for acute myelogenous leukaemia comprising adriamycin, cytarabine, and thioguanine does not necessarily cause azoospermia. By contrast, treatment for acute non-myelogenous leukaemia (induction of remission with adriamycin, vincristine, prednisolone, and colaspase followed by cranial irradiation and then three years' maintenance treatment with cyclophosphamide, mercaptopurine, and methotrexate) usually does.

Differences in both the schedule and the individual drugs used may have accounted for these findings. The hormonal studies suggest that sterility results from gonadal failure rather than from pituitary dysfunction after cranial irradiation. The most likely causal agent, absent from the treatment given for acute myelogenous leukaemia, is cyclophosphamide."

The finding of gonadal failure as a consequence of successful treatment of acute lymphatic or undifferentiated leukaemia and the observation of normal spermatogenesis in men treated for acute myeloid leukaemia have important implications for the counselling of patients with leukaemia.

Miss Jane Moore typed the manuscript. We thank Mr Les Perry for the hormone assays, and the patients for participating in the study.

\section{References}

${ }^{1}$ Fattah DI, Chard T. Method for measuring sex hormone binding globulin. Clin Chem $1981 ; 27: 1277-9$

2 Fairley KF, Barrie JU, Johnson W. Sterility and testicular atrophy related to cyclophosphamide therapy. Lancet 1972 ; :568-9.

(Accepted 4 August 1983)

ONE HUNDRED YEARS AGO The recent New York legislation gives a man very complete far-reaching and apparently retrospective rights to possess himself in pieces as well as in peace, and under the most adverse circumstances. "A person," says section 305 , "has the right to direct the manner in which his body shall be disposed of after his death, and to direct the manner in which any part of his body which becomes separated therefrom during his life-time shall be disposed of." Such claims might, it is thought in America, seriously affect the pathological museums of the hospitals, for which dissevered and morsels of humanity have hitherto been appropriated without legal leave asked or permission granted. It is feared that some fortunate possessors of rare pathological growths may set an unreasonably high value on their morbid peculiarities, when once they discover that they are their legal property and have a marketable value. A man's bones may become of more worth than himself, and his little finger of more value than all the rest of his body. (British Medical fournal $1883 ;$; :167.)

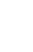

(1)

